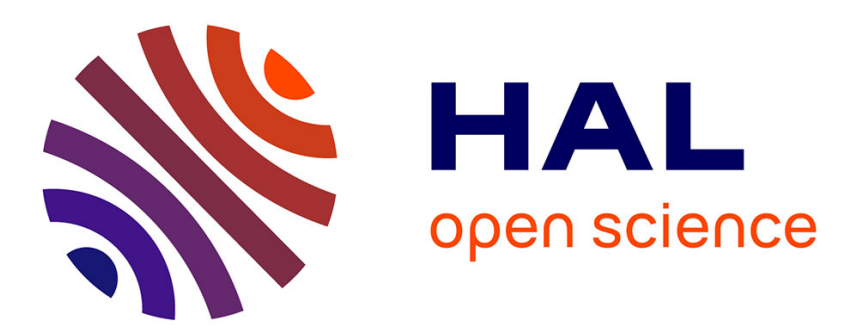

\title{
Interconnection and Damping Assignment - Passivity Based Control of Irreversible Port Hamiltonian Systems.
}

\author{
Héctor Estay, Yann Gorrec, Bernhard Maschke
}

\section{To cite this version:}

Héctor Estay, Yann Gorrec, Bernhard Maschke. Interconnection and Damping Assignment - Passivity Based Control of Irreversible Port Hamiltonian Systems.. 19th World Congress of the International Federation of Automatic Control, IFAC'14., 2014, Cape Town, South Africa. pp.9111-9116. hal01324732

\section{HAL Id: hal-01324732 \\ https://hal.science/hal-01324732}

Submitted on 1 Jun 2016

HAL is a multi-disciplinary open access archive for the deposit and dissemination of scientific research documents, whether they are published or not. The documents may come from teaching and research institutions in France or abroad, or from public or private research centers.
L'archive ouverte pluridisciplinaire HAL, est destinée au dépôt et à la diffusion de documents scientifiques de niveau recherche, publiés ou non, émanant des établissements d'enseignement et de recherche français ou étrangers, des laboratoires publics ou privés. 


\title{
Interconnection and Damping Assignment - Passivity Based Control of Irreversible Port Hamiltonian Systems
}

\author{
Hector Ramirez* Yann Le Gorrec* Bernhard Maschke** \\ * Department of Automation and Micro-Mechatronic Systems, \\ FEMTO-ST UMR CNRS 6174, ENSMM, 26 chemin de l'épitaphe, \\ F-25030 Besançon, France. \{ramirez, legorrec\}@femto-st.fr \\ ** Université de Lyon, Lyon, F-69003, France ; Université Lyon 1, \\ Faculté Sciences et Technologie, France ; CNRS, UMR500\%, \\ Laboratoire d'Automatique et Génie des Procédés, Villeurbanne, \\ F-69622, France (e-mail:\{ramirez,maschke\}@lagep.univ-lyon1.fr)
}

\begin{abstract}
Irreversible port Hamiltonian systems are a class of pseudo Hamiltonian systems that expresses both the conservation of energy and the irreversible entropy production as a structural property. These systems encompass a large class of irreversible themordynamic systems, such as heat exchangers and chemical reactors, and also multi-energy systems such as coupled mechanicthermodynamic systems. In recent work the irreversible port-Hamiltonian formulation has been used to derive a closed-loop stability condition using an energy based availability function, generated by the internal energy, as Lyapunov function. This paper presents an important extension of the previous results: the system theoretic interpretation of the stability condition in terms of conjugated inputs and outputs and the formulation of the control as an interconnection and damping assignment - passivity based control problem. A constructive method to derive the stabilizing control law is proposed and the formalism is illustrated on a general CSTR example.
\end{abstract}

Keywords: Irreversible port Hamiltonian systems, Passivity based control, Irreversible thermodynamics, Entropy creation, CSTR.

\section{INTRODUCTION}

The attempts to model irreversible processes as PHS have lead to a class of system called quasi PHS (Hangos et al., 2001; Otero-Muras et al., 2008; Ramirez et al., 2009; Dörfler et al., 2009; Hoang et al., 2011). These systems retain much of the port Hamiltonian structure, but differ by their structure matrices and input vector fields which depend explicitly on the gradient of the Hamiltonian. An important remark is that, although the forms of PHS and quasi PHS are very similar and both embed, by skew-symmetry of the structure matrix, the conservation of the Hamiltonian, in the latter the drift dynamic is a nonlinear function in the gradient $\frac{\partial U}{\partial x}(x)$. In this sense the symplectic structure of the PHS, given by the Poisson tensor associated with the structure matrix $J(x)$, is destroyed. From a control perspective it is usually more complicated to impose a desired closed-loop dynamic on quasi PHS since passivity based techniques can not be easily applied due to the non-linearity with respect to the gradient of the Hamiltonian.

In Ramirez et al. (2013b) a class of quasi PHS, denoted Irreversible Port Hamiltonian Systems (IPHS), has been proposed to model a large class of thermodynamic systems and to embed the first (conservation of the Hamiltonian)

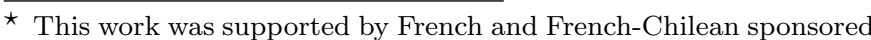
projects HAMECMOPSYS ANR-11-BS03-0002, Labex ACTION ANR-11-LABX-01-01 and ECOS-CONICYT C12E08.
}

and the second principle (irreversible increase of an entropy like function). IPHS have two important properties: they provide an irreversible non-linear system a physically meaningful structure (just as PHS for mechanical systems); and they are defined with respect to the total energy, which makes it possible to interconnect them with reversible non-linear systems (mechanical systems) (Ramirez et al., 2013c), which is not the case for formulations defined with respect to the total entropy for instance.

In Ramirez et al. (2013a) the framework of IPHS has been combined with the framework of thermodynamic availability function to derive an asymptotic stability condition for irreversible thermodynamic systems. Indeed, since the closed-loop equilibrium differs from the openloop equilibrium it is necessary to perform the closed-loop stability analysis with respect to a modified Hamiltonian, which in our case is derived departing from the total energy (open-loop Hamiltonian). In this paper we specialize the previous result for input-affine IPHS (Section 2) and interpret the stability condition in terms of interconnection and damping assignment - passivity based control (Ortega et al., 2001, 2002) (Section 3). It is interesting to see that the closed-loop system can again be interpreted as an irreversible thermodynamic systems with respect to a modified thermodynamic potential (Section 3). A constructive method to derive the stabilizing control law is proposed and the formalism is illustrated on a general 
CSTR example (Section 4). Finally (Section 5), some closing remarks and comments on future work are given.

\section{IRREVERSIBLE PORT-HAMILTONIAN SYSTEMS}

Irreversible Port Hamiltonian Systems (IPHS) have been defined in Ramirez et al. (2013b) as a modification of Port Hamiltonian systems (PHS) and allow to represent simultaneously the conservation of energy and the entropy creation due to an irreversible processes. An IPHS uses two thermodynamic potentials in order to define the dynamics: the total energy and the total entropy of a thermodynamic system and is defined as follows.

Definition 1. An input affine IPHS is defined by the dynamic equation

$$
\begin{aligned}
& \dot{x}=R\left(x, \frac{\partial U}{\partial x}, \frac{\partial S}{\partial x}\right) J \frac{\partial U}{\partial x}(x)+g\left(x, \frac{\partial U}{\partial x}\right) u, \\
& y=g^{\top}\left(x, \frac{\partial U}{\partial x}\right) \frac{\partial U}{\partial x}(x)
\end{aligned}
$$

where $x \in \mathbb{R}^{n}$ is the state vector, $U(x): \mathcal{C}^{\infty}\left(\mathbb{R}^{n}\right) \rightarrow \mathbb{R}$ is the Hamiltonian function (for thermodynamic systems, the internal energy) and $S(x): \mathcal{C}^{\infty}\left(\mathbb{R}^{n}\right) \rightarrow \mathbb{R}$ is a real function (for thermodynamic systems the total entropy function), $J \in \mathbb{R}^{n \times n}$ is the constant skew-symmetric structure matrix of the Poisson bracket acting on any two $C^{\infty}\left(\mathbb{R}^{n}\right)$ functions $Z$ and $G$ as:

$$
\{Z, G\}_{J}=\frac{\partial Z^{\top}}{\partial x}(x) J \frac{\partial G}{\partial x}(x) .
$$

The drift term of the system (1) differs from a Hamiltonian system by the multiplication by the real function $R=$ $R\left(x, \frac{\partial U}{\partial x}, \frac{\partial S}{\partial x}\right)$. This function is composed of the product of a positive definite function $\gamma$ and the Poisson bracket of the entropy function $S(x)$ and the energy function $U(x)$ :

$$
R\left(x, \frac{\partial U}{\partial x}, \frac{\partial S}{\partial x}\right)=\gamma\left(x, \frac{\partial U}{\partial x}\right)\{S, U\}_{J},
$$

with $\gamma\left(x, \frac{\partial U}{\partial x}\right)=\hat{\gamma}(x): \mathcal{C}^{\infty}\left(\mathbb{R}^{n}\right) \rightarrow \mathbb{R}, \hat{\gamma} \geq 0$, a nonlinear positive function. The input map is defined by $g\left(x, \frac{\partial U}{\partial x}\right) \in \mathbb{R}^{n \times m}$ with the input $u(t) \in \mathbb{R}^{m}$ a time dependent function.

The balance equations of the entropy function $S(x)$ and the energy function $U(x)$ of the IPHS express the first and second principles of the irreversible thermodynamics: the conservation of energy and the irreversible entropy creation due to the irreversible processes. Indeed consider the balance equation of the internal energy, one computes

$$
\frac{d U}{d t}=y^{\top} u
$$

where $u(t)$ and $y(z)$ are the input and output port of the system. This is precisely the balance equation corresponding to lossless dissipative systems with the (energy) supply rate $y^{\top} u$. The balance equation of the entropy function is given by

$$
\begin{aligned}
\frac{d S}{d t} & =R\left(x, \frac{\partial U}{\partial x}, \frac{\partial S}{\partial x}\right) \frac{\partial S^{\top}}{\partial x} J(x) \frac{\partial U}{\partial x}+\frac{\partial S^{\top}}{\partial x} g\left(x, \frac{\partial U}{\partial x}\right) u \\
& =\gamma\left(x, \frac{\partial U}{\partial x}\right)\{S, U\}_{J}^{2}+\left(g^{\top}\left(x, \frac{\partial U}{\partial x}\right) \frac{\partial S}{\partial x}\right)^{\top} u
\end{aligned}
$$

From the assumptions defining the IPHS (Definition 1) the first term is positive: $\gamma\left(x, \frac{\partial U}{\partial x}\right)\{S, U\}_{J}^{2}=\sigma\left(x, \frac{\partial U}{\partial x}\right) \geq 0$ which represents for models of irreversible thermodynamic systems, the internal entropy production. The second term corresponds to the definition of an entropy supply rate. For further details on IPHS and its thermodynamic interpretation we refer the reader to Ramirez et al. (2013b).

\section{IDA-PBC FOR IPHS}

In Ramirez et al. (2013a) the framework of IPHS and available storage (Willems, 1972) has been used to propose a class of PBC for irreversible thermodynamic systems. Using the convexity of the internal energy, the Hamiltonian function of an IPHS has been used as convex extension to define an energy based availability function which serves as Lyapunov function for the closed-loop system. This has been done using the same thermodynamic arguments as for instance in Alonso and Ydstie (1996); Ydstie and Alonso (1997); Alonso and Ydstie (2001); Ydstie (2002); Hoang et al. (2011, 2012), being the difference that in our case the closed-loop Lyapunov function is generated by the energy of the system and not the entropy. This permits to state the control problem similarly as for mechanical systems, with the difference that the irreversible nature of IPHS introduces an "interconnection obstacle" (Ramirez et al., 2013c), also refereed as "dissipation obstacle" in the case of dissipative PHS (Ortega et al., 2001). In this section we shall elaborate on the IDA-PBC method for deriving a stabilizing control for which the closed-loop Hamiltonian function is the energy-based availability function, i.e., we will investigate the existence of controls that renders the closed-loop system into the form

$$
\dot{x}=\mathcal{M}(x) \frac{\partial A}{\partial x}(x)
$$

where $A$ is the energy-based availability function and $\mathcal{M}(x)$ the closed-loop structure matrix, possibly defining an (dissipative) IPHS.

\subsection{The Energy based availability function}

Let us briefly recall some fundamental properties of irreversible homogeneous thermodynamic systems and more precisely the notion of the thermodynamic availability function associated with the internal energy (Ramirez et al., 2013c). The variation of the internal energy of an homogeneous system is defined by Gibbs' fundamental equation on a $N$-dimensional state space:

$$
d U=T d S-P d V+\sum_{i=1}^{N-2} \mu_{i} d n_{i}
$$

where the extensive variables are the internal energy $U$, the entropy $S$, the volume $V$ and the mole numbers $n_{i}$, and the intensive variables are the temperature $T$, the pressure $P$ and the chemical potentials $\mu_{i}$. By defining the vectors of extensive and intensive variables, $z=\left[S, V, n_{1}, \ldots, n_{N-2}\right]^{\top}$ and $w(z)=$ $\left[T(z),-P(z), \mu_{1}(z), \ldots, \mu_{N-2}(z)\right],(3)$ may be written as

$$
d U=w(z)^{\top} d z .
$$

The internal energy $U$ is a homogeneous function of degree 1, so from Euler's Theorem we obtain,

$$
U=w(z)^{\top} z,
$$

which implies that $w(z)$ is a homogeneous function of degree 0 of $z$ and $w(z)=\frac{\partial U}{\partial z}(z)$. For homogeneous systems, 
as a consequence of the second law of Thermodynamics (Callen, 1985; Alonso and Ydstie, 2001), the internal energy corresponds to a convex function. Furthermore the internal energy function is strictly convex with respect to $z$ and independent of the dynamic behaviour of the system if one of the extensive variables, i.e. one of the elements in $z$, is fixed (Jillson and Ydstie, 2007). For thermodynamic systems, the total energy function (and hence also the total entropy function) only possesses a strict minimum at the thermodynamic equilibrium, which does not coincide with the desired dynamic equilibrium. Indeed, at thermodynamic equilibrium the system does not undergo any irreversible transformation, hence it is not of practical interest.

Since the Hamiltonian of IPHS is the total energy it is natural to derive a closed-loop Lyapunov function using the convexity of the total energy, rather than the concavity of the total entropy. Recalling Definition 1, and taking $x$ as the state vector of the IPHS of we give the definition of the energy based availability function and the closed-loop stability condition.

Definition 2. The energy based availability function is given by

$$
A\left(x, x^{*}\right)=U(x)-\left[U\left(x^{*}\right)+\frac{\partial U}{\partial x}\left(x^{*}\right)^{\top}\left(x-x^{*}\right)\right] \geq 0
$$

where $x^{*}$ is a reference and possibly a desired equilibrium. For a thermodynamic system $A$ becomes a strictly convex with minimum value $A=0$ if one of the extensive variables is fixed (Jillson and Ydstie, 2007), i.e., if $z \in \mathbb{R}^{N-1}=\mathbb{R}^{n}$ and the state space is chosen as $x=z$. Furthermore, the only possible value for which $A=0$ is $x=x^{*}$.

The reader is refereed to Alonso and Ydstie (1996); Ydstie and Alonso (1997); Alonso and Ydstie (2001); Ydstie (2002) for further details on the construction and thermodynamic properties of the availability function. It is clear from Definition 2 that the energy based availability function qualifies as a Lyapunov function candidate for controlled IPHS.

Proposition 1. The closed-loop equilibrium $x^{*}$ of a controlled IPHS is asymptotically stable if $A$ is strictly convex and the control $u(x)$ satisfies:

$$
\gamma\{S, U\}_{J}\{A, U\}_{J}+\left(\frac{\partial U}{\partial x}(x)-\frac{\partial U}{\partial x}\left(x^{*}\right)\right)^{\top} g u \leq 0,
$$

with strict equality only at $\left(x^{*}, u^{*}\right)$, the steady state value at the desired equilibrium.

Proof. The time derivative of $A$ is given by

$$
\frac{d A}{d t}=\left(\frac{\partial U}{\partial x}(x)-\frac{\partial U}{\partial x}\left(x^{*}\right)\right)^{\top} \frac{d x}{d t} .
$$

Using Definition 1 we obtain the balance equation of the availability function

$$
\begin{aligned}
\frac{d A}{d t}= & -R\left(\frac{\partial U}{\partial x}\left(x^{*}\right)^{\top} J \frac{\partial U}{\partial x}(x)\right)+ \\
& \left(\frac{\partial U}{\partial x}(x)-\frac{\partial U}{\partial x}\left(x^{*}\right)\right)^{\top} g u, \\
= & \gamma\{S, U\}_{J}\{A, U\}_{J}+ \\
& \left(\frac{\partial U}{\partial x}(x)-\frac{\partial U}{\partial x}\left(x^{*}\right)\right)^{\top} g u,
\end{aligned}
$$

Notice that $\{A, A\}_{J^{\prime}}=0$ and does not contribute in (13). This bracket is included however since it shows that the closed-loop vector field may be shaped without injecting more damping into the system. This is referred to as the interconnection assignment. Now, using the definition of $\tilde{y}$ (Eq. (11)) we have that the unique solution to (14) is 


$$
\begin{aligned}
\beta(x)= & \\
g^{\dagger}(x)\left(-\{S, U\}_{J} M(x)+J^{\prime}(x)\right) & \left(\frac{\partial U}{\partial x}(x)-\frac{\partial U}{\partial x}\left(x^{*}\right)\right) \\
& -g^{\dagger}(x) J \frac{\partial U}{\partial x}(x), \quad(16)
\end{aligned}
$$

which exists if and only if

$$
\begin{aligned}
&-g^{\perp}(x)\{S, U\}_{J} M(x)\left(\frac{\partial U}{\partial x}(x)-\frac{\partial U}{\partial x}\left(x^{*}\right)\right) \\
&+g^{\perp}(x)\left(\left(J^{\prime}-J\right) \frac{\partial U}{\partial x}(x)-J^{\prime} \frac{\partial U}{\partial x}\left(x^{*}\right)\right)
\end{aligned}
$$

with $g^{\dagger}(x)=\left[g^{\top}(x) g(x)\right]^{-1} g^{\top}(x)$ the Moore-Penrose pseudo inverse and $g^{\perp}(x)$ a left full rank annihilator of $g(x)$, i.e., $g^{\perp}(x) g(x)=0$. Equations (16 and (17) express the well known IDA-PBC problem, but for irreversible control system.

Let us compare more in details this solution with the solution for a reversible port-Hamiltonian system

$$
\dot{x}=J \frac{\partial U}{\partial x}+g \tilde{u}
$$

keeping the same target system corresponding to the equivalent IDA-PBC problem defined by equations (16) and (17). The target system is then given by

$$
\dot{x}=\left(-\{S, U\}_{J} M(x)+J^{\prime}(x)\right) \frac{\partial A}{\partial x},
$$

where the matrix $\{S, U\}_{J} M(x)$ is not necessary positive semi-definite, since $\{S, U\}_{J}$ is of arbitrary sign. This "contradiction" comes of course from the definition of the control input, $u=\gamma\{S, U\}_{J} \tilde{u}$, but it also expresses the irreversible nature of the control system. The system is already dissipative, hence damping injecting is not the issue. The issue is to make the closed-loop system dissipate in such a way that it is stable. This contradiction has been reported in the literature as the "dissipation obstacle" for reversible PHS (Ortega et al., 2001), and more recently, as the "interconnection obstacle" for IPHS (Ramirez et al., 2013c). The previous results can be summarized as follows.

Proposition 2. Consider the input affine IPHS (1). Assume $A$ is strictly convex and that there exist matrices $M(x) \geq 0$ and $J^{\prime}(x)=-J^{\prime \top}(x)$, and a full-rank left annihilator $g^{\perp}(x)$ of $g(x)$ that verify (17). Then the control $u=\gamma\{S, U\}_{J} \beta(x)$, with $\beta(x)$ defined as in (16), asymptotically stabilizes the closed-loop equilibrium $x^{*}$. Furthermore, the closed-loop system is given by the pseudo-PHS

$$
\dot{x}=\left(-\sigma M(x)+R J^{\prime}(x)\right) \frac{\partial A}{\partial x} .
$$

Proof. The proof follows directly from the previous developments and (20) is obtained by replacing the control law $u=\gamma\{S, U\}_{J} \beta(x)$ in (1).

In some cases it is possible to use the physical properties of a system to solve (17). For instance, if we don't want to change the open-loop structure/interconnection matrix, and only express the natural dissipation of the system with respect to $A$, then the simplest choice is some constant $M>0$ and $J^{\prime}=J$.

Corollary 3. Assume $J^{\prime}=J$, then the closed-loop equilibrium $x^{*}$ is asymptotically stable if

$$
\begin{aligned}
g^{\perp}(x) J \frac{\partial U}{\partial x}\left(x^{*}\right) & =0, \quad \text { and } \\
g^{\perp}(x) M(x) & =0 .
\end{aligned}
$$

The second condition of Corollary 3 is in general easy to fulfil since the only restriction on $M$ is that it is positive semi-definite and symmetric. The first condition is however very restrictive, since $J, U(x)$ and $g(x)$ (and hence also $g^{\perp}(x)$ ) are defined by the physical system. Nevertheless, since $J$ expresses the conserved quantities of the thermodynamic process we may find this condition automatically fulfilled for systems whose inputs are related with the physical invariants. This point will be illustrated on the example of the CSTR.

\section{EXAMPLE: THE CSTR}

Assume a chemical reaction in a CSTR with the following reversible reaction scheme

$\nu_{1} B_{1}+\ldots+\nu_{l} B_{l} \rightleftharpoons \nu_{l+1} B_{l+1}+\ldots+\nu_{m} B_{m}, \quad m>l \geq 1$.

where where $B_{i}$ denotes the $i$ th chemical species, $\bar{\nu}_{i}$ is the signed stoichiometric coefficient: $\bar{\nu}_{i}=-\nu_{i}$ if it appears on the left hand side of the reaction scheme, $\bar{\nu}_{i}=\nu_{i}$ in the other case. We shall consider that the CSTR satisfies the following standard operation assumptions (Aris, 1989; Favache and Dochain, 2009)

Assumption 4.

- The volume, denoted by $V$, in the reactor is constant as well as the pressure,

- The initial number of moles of a specie in the reactor is equal to the number of moles of the inlet of the same specie,

- For a given steady state temperature and steady state input there is only one possible steady state for the mass (numbers of moles) balance.

- The reactor operates at gas phase,

- The controlled inputs are the the dilution rate $u_{1}=$ $F / V$ and the heat flux from the cooling jacket $u_{2}=Q$.

We have assumed a reaction in gas phase, but the developments may be applied identically to a reactor with a reaction in liquid phase. The assumptions of constant volume and pressure impose a constraint over the total outlet flow (Couenne et al., 2006, 2008), making the outlet flows state dependent. Furthermore this assumption guarantee that the energy based availability function $A$ is strictly convex.

The mass balance equations The time variation of the species in the reactor are given by (Aris, 1989)

$$
\dot{n}_{i}=\frac{F}{V}\left(n_{e i}-n_{i}\right)+r_{i} V \quad i=1, \ldots, m
$$

where $n_{i}$ and $n_{e i}$ are, respectively, the number of moles of the species $i$ in the reactor and at the inlet (and $\mathbf{n}=\left(n_{1}, \ldots, n_{m}\right)^{\top}$ and $\left.\mathbf{n}_{\mathbf{e i}}=\left(n_{e 1}, \ldots, n_{e m}\right)^{\top}\right), r_{i}=\bar{\nu}_{i} r$ where $r(\mathbf{n}, T)$ is the reaction rate which is the difference of the forward reaction rate $r_{f}$ and the backward reaction rate $r_{b}: r=\left(r_{f}-r_{b}\right)$ and depends on the temperature and on the reactant mole number and $F$ is the volumetric flow rate. 
The energy and entropy balance equations Under Assumption 4 the internal energy of the CSTR is given by (Couenne et al., 2006; Favache and Dochain, 2009)

$$
U=\sum_{i=1}^{m} n_{i}\left[c_{p i}\left(T-T_{0}\right)+u_{0 i}\right]
$$

where $c_{p i}, u_{0 i}, T_{0}$ are respectively the heat capacity at constant pressure, reference molar energy and reference temperature. The entropy balance equation may be deduced from Gibbs' relation and is given by

$$
\dot{S}=\frac{F}{V} \sum_{i=1}^{m}\left(n_{e i} s_{e i}-n_{i} s_{i}\right)+\frac{Q}{T_{e}}+\sigma,
$$

where $s_{e i}$ and $s_{i}$ are respectively the inlet molar entropy and the molar entropy of species $i, \sigma$ is the irreversible entropy creation due to mass transfer, heat transfer and chemical reactions:

$$
\sigma=\frac{F}{V} \sum_{i=1}^{m} \frac{n_{e i}}{T}\left(h_{e i}-T s_{e i}-\mu_{i}\right)+\frac{Q}{T}-\frac{Q}{T_{e}}-\sum_{i=1}^{m} \mu_{i} \nu_{i} \frac{r}{T},
$$

where $Q=\lambda\left(T_{e}-T\right)$ is the heat flux from the jacket with $\lambda$ the heat conduction coefficient, $T_{e}$ the temperature of the jacket, $h_{e i}$ the inlet specific molar enthalpy.

IPHS model (Ramirez et al., 2013b) The derivation of the IPHS model of the CSTR has been presented in details in Ramirez et al. (2013b). The dynamical equation of the CSTR may be expressed as the IPHS

$$
\dot{x}=R J \frac{\partial U}{\partial x}(x)+g\left(x, \frac{\partial U}{\partial x}\right) u
$$

with state vector $x=\left[\mathbf{n}^{\top}, S\right]^{\top}$, the internal energy $U(x)$ as Hamiltonian function,

$$
J=\left[\begin{array}{cccc}
0 & \ldots & 0 & \bar{\nu}_{1} \\
0 & \ldots & 0 & \vdots \\
0 & \ldots & 0 & \bar{\nu}_{m} \\
-\bar{\nu}_{1} & \ldots & -\bar{\nu}_{m} & 0
\end{array}\right]
$$

a constant skew-symmetric matrix whose elements are the stoichiometric coefficient of the chemical reaction mapping the network structure of the reaction, and

$$
R=\gamma\left(x, \frac{\partial U}{\partial x}\right)\{S, U\}_{J}=\left(\frac{r V}{T \mathcal{A}}\right) \mathcal{A}
$$

with $\gamma=\frac{r V}{T \mathcal{A}}$ and $\{S, U\}_{J}=\mathcal{A}$, where $\mathcal{A}=-\sum_{i=1}^{m} \bar{\nu}_{i} \mu_{i}$ is the chemical affinity of the reaction and corresponds to the thermodynamic driving force of the chemical reaction. The input port of the IPHS is given by $g u$, with $u=\left[u_{1}, u_{2}\right]^{\top}$, where $u_{1}$ is the dilution rate $\frac{F}{V}$ and $u_{2}$ the heat flux from the cooling jacket $Q$. The first row of the input map is the an input and output mole number vector and second row a thermal interaction vector,

$$
g=\left[\begin{array}{cc}
\tilde{\mathbf{n}} & \mathbf{0} \\
\phi(x) & \frac{1}{T}
\end{array}\right]
$$

with $\tilde{\mathbf{n}}=\mathbf{n}_{\mathbf{e}}-\mathbf{n}$, and $\phi(x)=\sum_{i=1}^{m}\left(n_{e i} s_{e i}-n_{i} s_{i}\right)+\frac{n_{e i}}{T}\left(h_{e i}-\right.$ $\left.T s_{e i}-\mu_{i}\right)$.

\subsection{IDA-PBC of the CSTR}

In this subsection we present how to use the IPHS model of the CSTR (open-loop model) to derive a stabilizing control law which imposes a desired IPHS structure on the closed-loop system. We will use Corollary 3 to show that the state feedback 16 asymptotically stabilizes the CSTR at a desired equilibrium point $x^{*}$. For this purpose we select $J^{\prime}=J$ and should find a matrix $M$ such that (22) is satisfied. The first step is to find a full rank left annihilator for the input map. One possible choice is the $(m-1) \times n$ matrix

$$
g^{\perp}(x)=\left[\begin{array}{ccccccc}
\tilde{n}_{2} & -\tilde{n}_{1} & 0 & \ldots & 0 & 0 & 0 \\
0 & \tilde{n}_{3} & -\tilde{n}_{2} & \ldots & 0 & 0 & 0 \\
0 & 0 & \ddots & \ddots & \ddots & 0 & 0 \\
0 & 0 & \ldots & 0 & \tilde{n}_{m} & -\tilde{n}_{m-1} & 0
\end{array}\right]
$$

The most restrictive condition in Corollary 3 is (21), from which we obtain, when using the previously chosen annihilator,

$$
g^{\perp}(x) J=\left[\begin{array}{cccc}
0 & \ldots & 0 & \bar{\nu}_{1} \tilde{n}_{2}-\bar{\nu}_{2} \tilde{n}_{1} \\
0 & \ldots & 0 & \bar{\nu}_{2} \tilde{n}_{3}-\bar{\nu}_{3} \tilde{n}_{2} \\
\vdots & \vdots & \vdots & \vdots \\
0 & \ldots & 0 & \bar{\nu}_{m-1} \tilde{n}_{m}-\bar{\nu}_{m} \tilde{n}_{m-1}
\end{array}\right] .
$$

It suffices to verify that $g^{\perp}(x) J=0$, which is true if

$$
\frac{\tilde{n}_{1}}{\bar{\nu}_{1}}=\frac{\tilde{n}_{2}}{\bar{\nu}_{2}}=\cdots=\frac{\tilde{n}_{m-1}}{\bar{\nu}_{m-1}}=\frac{\tilde{n}_{m}}{\bar{\nu}_{m}} .
$$

From Assumption 4 we have that the initial numbers of moles of each specie equals the numbers of moles at the inlet, i.e., $\mathbf{n}(t=0)=\mathbf{n}_{0}=\mathbf{n}_{e}$. Hence, (28) is actually the expression of De Donder's extent of reaction (Callen, 1985)

$$
\frac{n_{0_{i}}-n_{i}}{\bar{\nu}_{i}}=\xi,
$$

which is always true and hence (21) also. It is interesting that (21) is automatically fulfilled for this example. This is due to that $J$ expresses the stoichiometry of the reaction and $g(x)$ the mole (mass) balance. Since the reactor operates at constant volume, the total mass becomes an invariant for the reaction, and $g^{\perp}(x) J=0$ is simply the mathematical expression of this invariant.

The second condition, given by (22), can be solved by noting that the last column of $g^{\perp}(x)$ is zero. This implies that any matrix $M(x)=M^{\top}(x)>0$ for which the first $m$ rows and columns form a null submatrix is solution to (21). A (simple) possible choice is $M=\operatorname{diag}(0, \ldots, 0,1)$, which corresponds to a matrix with all elements equal to zero except the last element of its diagonal which is 1 . The closed-loop system takes then the form

$$
\dot{x}=(-\sigma M+R J) \frac{\partial A}{\partial x}\left(x, x^{*}\right)
$$

and the time derivative of $A$ along the trajectories of the CSTR is

$$
\frac{d A}{d t}=-\sigma\left(T-T^{*}\right)^{2} .
$$

Hence, the "dissipation" of the energy based availability function is related to the irreversible energy production, which is positive definite and only zero at the thermodynamic equilibrium (which is not included in the region of operation of the CSTR). In order to complete the stability proof, we need to apply La Salle's invariance Theorem in a sufficiently small region around $T=T^{*}$. From Assumption 4 we have that there is only one equilibrium for each temperature, hence we can conclude that the closed-loop systems is asymptotically stable. 


\section{CONCLUSION}

The stabilization of irreversible thermodynamic systems has been studied using the framework of Irreversible portHamiltonian Systems (IPHS) and the thermodynamic availability function. The availability function is derived from the intrinsic properties of homogeneous thermodynamic systems, and under certain operation conditions can be used to analyse the dynamic properties, such as the stability, of a thermodynamic system, i.e., it may be used as a Lyapunov function for thermodynamic systems. In a first step, an energy based availability function has been defined and used to derive a general asymptotic stability condition for IPHS. It is indeed natural to use the energy, rather than the entropy, to derive the closed-loop availability function since the Hamiltonian of IPHS corresponds to the total energy. Then, in a second step, the stability condition has been developed an interpreted in terms of the matching of vector fields, leading to an Interconnection and Damping Assignment - Passivity Based Control (IDA$\mathrm{PBC}$ ) problem. The matching condition and the definition of the control law associated to the IDA-PB controller clearly expresses the irreversible nature of the open-loop system, and it is shown that the closed-loop target system is an dissipative IPHS with the energy based availability as Hamiltonian function. An IDA-PBC synthesis method for IPHS has been proposed and the control design approach has been illustrated on a general model of a chemical CSTR.

\section{REFERENCES}

Alonso, A.A. and Ydstie, B.E. (1996). Process systems, passivity and the second law of thermodynamics. Computers and Chemical Engineering, 20, 1119-1124.

Alonso, A.A. and Ydstie, B.E. (2001). Stabilization of distributed systems using irreversible thermodynamics. Automatica, 37, 1739-1755.

Aris, R. (1989). Elementary chemical reactor analysis. Chemical Engineering. Butterworths, Stoneham, USA.

Callen, H. (1985). Thermodynamics and an introduction to thermostatistics. Wiley, New-York.

Couenne, F., Jallut, C., Maschke, B., Breedveld, P., and Tayakout, M. (2006). Bond graph modelling for chemical reactors. Mathematical and Computer Modelling of Dynamical Systems, 12(2-3), 159-174.

Couenne, F., Jallut, C., Maschke, B., Tayakout, M., and Breedveld, P. (2008). Structured modeling for processes: A thermodynamical network theory. Computers $\&$ Chemical Engineering, 32(6), 1120 - 1134.

Dörfler, F., Johnsen, J., and Allgöwer, F. (2009). An introduction to interconnection and damping assignment passivity-based control in process engineering. Journal of Process Control, 19, 1413-1426.

Favache, A. and Dochain, D. (2009). Thermodynamics and chemical systems stability: The CSTR case study revisited. Journal of Process Control, 19, 371-379.

Hangos, K.M., Bokor, J., and Szederkényi, G. (2001). Hamiltonian view on process systems. AIChE Journal, 47, 1819-1831.

Hoang, H., Couenne, F., Jallut, C., and Le Gorrec, Y. (2011). The port Hamiltonian approach to modelling and control of continuous stirred tank reactors. Journal of Process Control, 21(10), 1449-1458.
Hoang, H., Couenne, F., Jallut, C., and Le Gorrec, Y. (2012). Lyapunov-based control of non isothermal continuous stirred tank reactors using irreversible thermodynamics. Journal of Process Control, 22(2), 412-422.

Jillson, K.R. and Ydstie, B.E. (2007). Process networks with decentralized inventory and flow control. Journal of Process Control, 17(5), 399 - 413.

Ortega, R., van der Schaft, A., Maschke, B., and Escobar, G. (2002). Interconnection and damping assignment passivity based control of port-controlled Hamiltonian systems. Automatica, 38, 585-596.

Ortega, R., van der Schaft, A., Mareels, I., and Maschke, B. (2001). Putting energy back in control. IEEE Control Systems Magazine, 21(2), 18-32.

Otero-Muras, I., Szederkényi, G., Alonso, A.A., and Hangos, K.M. (2008). Local dissipative Hamiltonian description of reversible reaction networks. Systems \& Control Letters, 57, 554-560.

Ramirez, H., Le Gorrec, Y., Maschke, B., and Couenne, F. (2013a). Passivity based control of irreversible portHamiltonian systems. In Proceedings of the 2013 IFAC Workshop on Thermodynamic Foundations of Mathematical Systems Theory (TFMST'13). Lyon, France.

Ramirez, H., Maschke, B., and Sbarbaro, D. (2013b). Irreversible port-Hamiltonian systems: A general formulation of irreversible processes with application to the CSTR. Chemical Engineering Science, 89(0), 223 - 234.

Ramirez, H., Maschke, B., and Sbarbaro, D. (2013c). Modelling and control of multi-energy systems: An irreversible port-Hamiltonian approach. European Journal of Control, 19(6), $513-520$.

Ramirez, H., Sbarbaro, D., and Ortega, R. (2009). On the control of non-linear processes: An IDA-PBC approach. Journal of Process Control, 19(3), 405 - 414.

Willems, J. (1972). Dissipative dynamical systems part I: General theory. Archive for Rational Mechanics and Analysis, 45, 321-351.

Ydstie, B.E. (2002). Passivity based control via the second law. Computers and Chemical Engineering, 26, 10371048.

Ydstie, B.E. and Alonso, A.A. (1997). Process systems and passivity via the clausius-planck inequality. Systems 8 Control Letters, 30(5), 253 - 264. 